dissimilarity index which indicate the percentage of the peak area of the fragment (operational taxonomic unit: OTU) was used.

Results Two fecal samples of $1 \mathrm{CC}$ infant and 14 fecal samples of 7 BA infants were obtained. Nine predominant OTUs were detected with Bsl I digestion. The microbiota consisted of microbial communities of Bifidobacterium, Lactobacillales, Bacteroides, Prevotella, Clostridium clusters IV, XI, and XVIII, and Clostridium subcluster XIVa. The Bifidobacterium, Bacteroides, and Clostridium clusters were detected predominatly in CC than BA group. Lactobacillales was most predominat group in BA feces.

Conclusions Bacterial DNA showed marked differences in the composition of fecal microbiota in CC and BA infants. Molecular analysis of colonic microbiota using $16 \mathrm{~S}$ rRNA gene libraries and T-RFLP might be useful to differentiate CC from BA.

\section{ASSESSMENT OF DNA DAMAGE USING COMET ASSAY AND DETECTION OF OXIDATIVE STRESS PARAMETERS IN DOWN SYNDROME}

doi:10.1136/archdischild-2012-302724.0734

${ }^{1} \mathrm{G}$ El-Saeed, ${ }^{2} \mathrm{~S}$ Abdel Maksoud. ${ }^{1}$ Medical Biochemistry; ${ }^{2}$ Clinical Pathology, National Research Center, Giza, Egypt

Background Down syndrome is one of the commonest numerical chromosomal aberrations. Recent studies showed that oxidative stress is an important pathological factor in Down syndrome.

Objective To estimate the level of oxidative stress and DNA damage in Down syndrome patients.

Patients and methods: Fifteen Egyptian patients clinically diagnosed and cytogenetically proven to have Down syndrome. Fifteen Egyptian healthy controls were recruited from the outpatient clinic of Clinical Genetics Department, National Research Centre. Oxidative stress parameters including total antioxidant capacity (TAC), Superoxide dismutase (SOD) enzyme activity and Malondialdehyde (MDA) biomarkers were estimated. DNA damage was determined using the alkaline comet assay.

Results The MDA and SOD levels in Down syndrome patients were significantly higher than control group $(p=0.000 \& 0.01$, respectively). Total antioxidants levels were non-significantly higher than control group $(p=0.54)$. Statistical analysis of DNA damage levels in DS patients compared to controls showed significant increased levels $(p=0.000)$. There was a positive correlation of DNA damage levels with age in DS patients but not reaching a significant value $(\mathrm{p}=0.536)$. A non-significant positive correlation was detected between DNA damage levels and both MDA and TAC levels ( $p=0.8 \& 0.37$, respectively). Also a non-significant negative correlation of DNA damage levels with SOD levels was noticed $(\mathrm{p}=0.14)$.

Conclusion Oxidative stress plays a major role in DS pathogenesis.

\section{CONTRIBUTION OF ALKYLATING AGENTS IN THE CYTOGENETIC DIAGNOSIS OF FANCONI ANEMIA}

doi:10.1136/archdischild-2012-302724.0735

S Bouraoui, S Mougou, 0 Mamai, H Elghezal, A Saad. Department of Cytogenetic and Reproductive Biology, Farhat Hached University Teaching Hospital, Sousse, Tunisia

Background and Aims Fanconi Anemia (FA) is an autosomal recessive disease characterized by heterogenous phenotype which includes a bone marrow failure, diverse abnormalities and increased predisposition to develop leukemia. The cytogenetic diagnosis of FA cells to bifunctional alkylating agents, resulting in greatly increased chromosomal breakage and radial stuctures induced by cross-linking agents. To estimate the sensitivity and the specificity of the Mitomicyn C (MMC) and the Diepoxybutan (DEB), two alkylating agents used in the diagnosis of the FA, we studied the chromosomal instability on 22 patients using variable types and concentrations of these alkylating ( 25 and $40 \mathrm{ng} / \mathrm{ml}$ of MMC, $0.1 \mu \mathrm{g} / \mathrm{ml}$ of DEB).

Methods Heparanized venous blood samples were collected and were processed for the cytogenetic methodology in this study. After culture, 100 of metaphases were analysed to evaluate the frequency of chromosomal aberrations.

Results The MMC test at $25 \mathrm{ng} / \mathrm{ml}$ was High sensitive for FA. The DEB test showed a better specificity. The study of the mitotic segregation of sexual chromosomes by FISH took away any abnormality of the segregation to cells FA.

Conclusions A molecular study of the sensivity and the specificity of the alkylating agents used according to the group of complementation will come refine the diagnosis of FA by establishing a gold standard.

\section{CLINICAL AND CYTOGENETIC STUDY IN 116 CHILDREN COHORT WITH SEXUAL AMBIGUITY}

doi:10.1136/archdischild-2012-302724.0736

'I Ben Hadj Hmida, 'S Dimassi, 'M Kammoun, ${ }^{2} \mathrm{~K}$ Ben Helal, ${ }^{1} \mathrm{H}$ Hannechi, ${ }^{3} \mathrm{H}$ Seboui, 'M Gribaa, 'S Mougou-Zerelli, 'A Saad. 'Departments of Cytogenetics and Reproductive Biology, Farhat Hached University Teaching Hospital; 2 Departments of Pediatic, Hospital Ibn El Jazar Kairouan; ${ }^{3}$ Departments of Neonatology, Farhat Hached University Teaching Hospital, Sousse, Tunisia

Disorder of sex development may result from multiple etiologies, led to many physical impacts involving genital abnormalities. Differentiation of a testis or an ovary from a bipotential gonad is a complex developmental process where various genes and hormones interact. Thus, the causality of sexual ambiguity was supported by the presence of many chromosomal abnormalities, especially when sex determination genes were involved.

A careful clinical examination will primarily interest to define the sexual ambiguity Prader score and the whole genital and extra genital malformation context.

Our study focuses on cases referred to the department of Cytogenetics and Reproductive Biology CHU Farhat Hached for ambiguous genitalia cytogenetic exploration from January 2010 to February 2012.

The 116 patients studied with ambiguous genitalia of varying severity, $80 \%$ were younger than 10 years. $7.5 \%$ of them showed chromosomal abnormalities.

In 3 patients, the chromosomal abnormality involved the autosomes 4, 5 and 1 with a $4 p$ deletion in Wolf-Hirschhorn syndrome, deletion $5 p$ in Cri-du-chat syndrome and $1 q$ deletion revealed by Comparative Genomic Hybridization-array. Theses anomalies are associated with loss of genital differentiation protein function. In 4 other patients abnormal sexual chromosome number was identified. For 86 children with normal karyotypes 46, XY, SRY gene molecular cytogenetic screening was performed. A deletion may in these cases explain the phenotype.

Identification of gene rearrangements causing urogenital birth defects can have an impact on the diagnosis and genetic counseling. Thus more study is necessary to find new candidate genes in signaling pathways regulating sexual differentiation.

\section{MOLECULAR CYTOGENETIC AND COMPARATIVE GENOMIC HYBRIDIZATION ANALYSIS IN NEWBORNS WITH CONGENITAL HEART DISEASES}

doi:10.1136/archdischild-2012-302724.0737

'I Malek, 'H Ben Khelifa, "I Ben Abdallah, 'H Hannen, 'S Mougou-Zerelli, ${ }^{2} \mathrm{H}$ Seboui, ${ }^{1} \mathrm{H}$ El Ghezal, 'A Saad. 'Departments of Cytogenetics and Reproductive Biology, Farhat Hached University Teaching Hospital; ' $N$ Neonatology Service, Farhat Hached University Teaching Hospital Sousse, Sousse, Tunisia

Congenital heart diseases (CHD) are abnormalities that occur at birth and resulting from a developmental defect during cardiac 
morphogenesis. Their prevalence is estimated between 0.4 and $0.6 \%$ of live births. CHD is essentially multifactorial. Among the genetic causes, chromosomal aberrations are involved in congenital heart disease. Indeed, among patients who carry chromosomal abnormalities, $30 \%$ have cardiovascular problems. $22 \mathrm{q} 11.2$ microdeletion is the most common cause.

The purpose of this study was to determine whether subtle chromosomal anomalies previously undetected by conventional cytogenetic banding methods could be identified by array-CGH in children with isolated CHD. We reported 30 unrelated newborns recruited from Neonatology service for genetic exploration.

Genetic investigations are essentially based on the techniques of cytogenetics and molecular cytogenetics. At first intension banded karyotyping was performed followed by fluorescent in situ hybridization (FISH) using gene-specific probe TUPLE1 in 22q11.2. As a last resort comparative genomic hybridization CGH-array 44K (Agilent ${ }^{\circledR}$ Technology) has been performed for 4 patients.

FISH showed normal hybridization to the DiGeorge syndrome critical region for all patients and no copy number variations was detected by array-CGH.

Our analysis was limited by a small and heterogenous study population. Also Increasing resolution arrays are needed to detect cryptic rearrangements.

We propose this strategy to explore a wider group of patients to identify new genetic factors involved in the development of cardiac malformations. The identification of genetic etiologies for CHD is important to provide genetic counseling and to establish a report genotype phenotype for every type of heart disorder.

\section{MOLECULAR CHARACTERIZATION OF DER (8) (0TER021.13: PTERP23.3) DN IN A CHILD ASSOCIATING PSYCHOMOTOR RETARDATION, HYDROCEPHALUS AND FACIAL DYSMORPHISM}

doi:10.1136/archdischild-2012-302724.0738

1.2 $\mathrm{H}$ Hannachi, ${ }^{1,2} \mathrm{~S}$ Mougou-Zerelli, ${ }^{3} \mathrm{~K}$ Ben Helal, 1,21 Ben Abdallah, 'D Hmida, 1.2 $\mathrm{H}$ Elghezal, ${ }^{1,2 \mathrm{~A}}$ Saad. 'Cytogenetic, Biologie Moléculaire et Biologie de la Reproduction Humaines, CHU Farhat Hached; '2Département Commun de Génétique, Université de Médicine, Sousse; ${ }^{3}$ Département de Pédiatrie, Hôpital Régional Iben el Jazzar, Kairouan, Tunisia

Complex but balanced chromosomal rearrangements can give rise, through recombination during meiosis, to complex unbalanced rearrangements. Here, we report on the case of a 21 months old child associating a 8q21.13 duplication and 8p23.3 microdeletion. The proposita was referred to our lab for cytogenetic exploration of a hydrocephalus associated with facial dysmorphism. He had also psychomotor retardation and microcephaly. The patient R-banding karyotype revealed a partial trisomy $8 \mathrm{q}$ captured by the $\mathrm{p}$ telomere of the same chromosome, whereas the parents' karyotypes were normal. CGH-array technique characterized breakpoints and estimated its size to $61.8 \mathrm{Mb}$. Interestingly, an additional cryptic loss of $260 \mathrm{~Kb}$ in 8(p23.3-pter) was also identified by the same technique. These anomalies were confirmed by FISH technique.

The partial deletion of a chromosome arm in combination with partial duplication of the other was evocative of a recombinant chromosome deriving from a parental pericentric inversion. We suggest, therefore that a parental pericentric prezygotic $8(\mathrm{p} 23.3-\mathrm{q}$ 21.13) inversion resulted in the complex unbalanced rearrangement of chromosome 8 in our patient. The clinical picture including hydrocephalus, inguinal hernia, long-term fever and psychomotor retardation, was described in patients with pure 8q2-qter duplication. However, the 8p23.3 microdeletion may contribute to the psychomotor retardation, microcephaly and some minor dysmorphic features. Here we emphasize the fact that the $8 p$ microdeletion would be cytogenetically undetectable in the absence of CGH-array technique underlining the need of entire genome high-resolution analysis in patients with idiopathic mental retardation and/or birth defects even in abnormal conventional karyotype cases.

\section{MONOSOMY: GENOTYPE-PHENOTYPE CORRELATION AND THERAPEUTIC IMPACT}

doi:10.1136/archdischild-2012-302724.0739

'M Kammoun, 'H Hannechi, 'H Hajlaoui, ${ }^{2} \mathrm{H}$ Soua, 'D Hmida, 'A Saad, 'S Mougou. ${ }^{1}$ Departments of Cytogenetics and Reproductive Biology, Farhat Hached University Teaching Hospital, Sousse, 2Pediatric Department, Taher Sfar Hospital, Mahdia, Tunisia

The deletion of the long arm of chromosome 18 causes a contiguous gene deletion syndrome with a highly variable phenotype, usually related to the extent of the deleted region. The most commonly reported clinical features include: mental disabilities, decreased growth, microcephaly and facial abnormalities.

We report on a case with partial monosomy 18q22 derived from a maternal reciprocal translocation $t(8 ; 18)$. The patient was 7 months old referred for genetic exploration of neurodevelopmental delay, craniofacial dysmorphism, post natal growth retardation, choanal atresia, club foot and congenital hip dislocation.

Chromosome analysis from peripheral blood showed a 46, XY, der (18). Array CGH was performed and revealed a partial monosomy $18 \mathrm{q} 21.33 \mathrm{q} 22.31$ of $15.3 \mathrm{MB}$ associated with partial trisomy $8 \mathrm{q} 24.12 \mathrm{q} 24.23$ of $22.7 \mathrm{MB}$ region. These results were confirmed by FISH using telomeric 18q probe.

Choanal atresia and skeletal malformation are in agreement with the monosomy 18q. Interestingly, the deletion includes GALR1 gene in 18q23 witch encodes galanine receptor. Galanine is a neuromodulator that stimulates growth hormone secretion. $M B P$, and adjacent genes, are implicated in myelination process and haploinsufficiency explains partially developmental delay. Otherwise, the haploinsufficiency of the 18q22.3-q23 gene region is suggested to be a critical region for the immunoglobuline A deficiency which is significantly associated to celiac disease. Our patient has not until now immunological desorders.

The association deletion 18q22- GH deficiency and decreased myelinisation is now well estabilished. The real therapeutic impact of GH treatment is discussed.

\section{DUPLICATION OF THE SOX3 GENE IN A SRY NEGATIVE 46 XX MALE}

doi:10.1136/archdischild-2012-302724.0740

'Z Gucev, ${ }^{2} \mathrm{~F}$ Riepe, ${ }^{3} \mathrm{~A}$ Gharavi, ${ }^{3} \mathrm{~S}$ Sanna-Cherchi, 'V Tasic. 'Medical Faculty Skopje, Skopje, FYR Macedonia; ${ }^{2}$ Clinic for General Pediatrics, Kiel, Germany; ${ }^{3}$ Division of Nephrology, Columbia University, New York, NY, USA

Case presentation An 11 old patient with hypoplasia of the right kidney and hypospadias was found to be SRY negative, 46, XX. His parents and younger sister were healthy. His intelligence was normal (IO 92) and he had no other anomalies. The behavior, growth and development were all normal. His testes were $>4 \mathrm{ml}$ and the penis was $5 \mathrm{~cm}$. Ultrasound and MRI did not show internal female genitals, while confirming right kidney hypoplasia (as did the DMSA scan).

ACTH test showed normal basal and stimulated $17 \mathrm{OH}$-progesterone excluding a form of 46XX DSD due to 21-hydroxylase deficiency. 11-DOC and 11S were normal at both baseline and after ACTH stimulation, excluding 11-hydroxylase deficiency. Cortisol levels were in the mid normal range at baseline and responded to stimulation, excluding primary adrenal insufficiency. Androstenedione,

The hCG test found testosterone in the low normal range for male sex and age at baseline. It rised up to $146 \mathrm{ng} / \mathrm{mL}$ indicating the presence of functional Leydig cells targeted by hCG. The stimulated ratio T:DHT was 5.6, not supporting 5 alpha-reductase deficiency. 